\title{
ESPONDILÓLISE E ESPONDILOLISTESE EM GINASTAS JOVENS
}

\author{
SPONDYLOLYSIS AND SPONDYLOLISTHESIS IN YOUNG GYMNASTS
}

Rafael Mohriak', Pedro Debieux Vargas Silva', Miguel Trandafilov Junior ${ }^{2}$, Délio Eulálio Martins ${ }^{3}$, Marcelo Wajchenberg ${ }^{3}$, Moisés Cohen ${ }^{4}$, Eduardo Barros Puertas ${ }^{5}$

\section{RESUMO}

Objetivo: Determinar a presença de espondilólise e espondilolistese em atletas de ginástica artística feminina e correlacionar com dor lombar, história de trauma e carga de treinamento. Método: Avaliação voluntária de 18 atletas de ginástica olímpica em nível competitivo de oito a 17 anos, com média de 11,3 anos. As ginastas responderam a um questionário em relação às suas atividades esportivas e foram submetidas a exame clínico e radiográfico da coluna lombar. Resultados: A análise das radiografias foi realizada por ortopedistas especialistas em cirurgia de coluna vertebral, obtendo prevalência de 5,56\% para espondilólise e não havendo casos de espondilolistese. Conclusão: A incidência das alterações radiográficas identificadas é semelhante à relatada na literatura para indivíduos não atletas e a lombalgia apontada pelas atletas não apresentou relação direta com a espondilólise ou espondilolistese.

Descritores - Ginástica; Coluna vertebral; Lombalgia; Espondilólise; Espondilolistese

\section{ABSTRACT}

Objective: To determine the incidence of spondylolysis and spondylolisthesis in young, female gymnasts, and to correlate these with lumbar pain, history of trauma and training load. Method: A total of 18 would-be Olympic gymnasts, aged 8-17, with an average age of 11.3, were voluntarily evaluated. The Gymnasts were asked to complete a questionnaire about their sports activities, and were submitted to a clinical examination and lumbar spine radiography. Results: The radiographies were analyzed by experienced specialists in surgery of the vertebral column, who found a $5.56 \%$ incidence of spondylolysis and no cases of spondylolisthesis. Conclusion: The incidence of radiographic alterations identified remained close to those reported in the literature for non-athletic individuals, and the lumbalgia indicated by the athletes showed no direct relation with spondylolysis and spondylolisthesis.

Keywords - Gymnastics; Spine; Lumbalgia; Spondylolysis; Spondylolisthesis

\section{INTRODUÇÃO}

“A ginástica olímpica é um esporte que congrega a arte da dança, a velocidade dos esportes radicais, a agilidade dos saltos ornamentais e a flexibilidade do balé”(1). As provas oficiais de ginástica olímpica (artística) diferem em relação ao sexo. No masculino existem seis provas (salto sobre o cavalo, exercícios de solo, cavalo com alça, argolas, barras paralelas, e barra fixa), enquanto que, no feminino, são quatro (salto sobre o cavalo, trave de equilíbrio, exercícios de solo e barras assimétricas).
O risco de lesão na prática desse esporte é o dobro do de qualquer outro esporte feminino se desconsiderarmos a gravidade do incidente ${ }^{(2-4)}$. A coluna vertebral responde por 17,2\% do total de lesões na ginástica olímpica, correspondendo à segunda sede mais frequente de acidentes $^{(3-7)}$. A espondilólise compromete $11 \%$ das ginastas do sexo feminino, local mais frequente é a quinta vértebra lombar e é caracterizada pela presença de dores lombares que pioram com a corrida e a queda e melhoram com o repouso e a flexão do tronco ${ }^{(1,8-10)}$. No exame físico dessas pacientes detectam-se hiperlordose lombar e encurtamento da musculatura posterior da $\operatorname{coxa}^{(1,9)}$.

1 - Médico Residente do Departamento de Ortopedia e Traumatologia da Escola Paulista de Medicina-Unifesp, São Paulo, Brasil.

2 - Médico Assistente do Grupo de Coluna do Departamento de Ortopedia e Traumatologia da Escola Paulista de Medicina-Unifesp, São Paulo, Brasil.

3 - Médico Assistente do Grupo de Coluna do Departamento de Ortopedia e Traumatologia da Escola paulista de Medicina-Unifesp, São Paulo, Brasil.

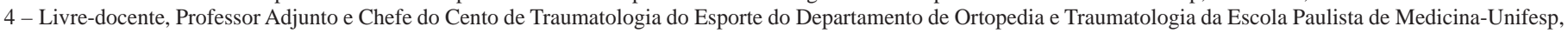
São Paulo, Brasil.

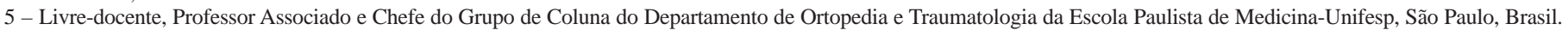

Trabalho realizado no Departamento de Ortopedia e Traumatologia do Hospital São Paulo, Escola Paulista de Medicina - Unifesp.

Correspondência: Rua Galleazzo Alessi, 70, ap. 81 - 04305-050 - São Paulo. E-mail: rmohriak@yahoo.com 
O mecanismo de lesão mais frequente é a hiperextensão em geral causada pelo impacto contra o solo na saída dos aparelhos ou mecanismo de torções durante os exercícios, tais como os movimentos em parafuso ${ }^{(9,10)}$. Outros fatores predisponentes da dor lombar são a hiperlordose e os desequilíbrios musculares ${ }^{(10)}$. Dentre as causas de lombalgia em atletas, a espondilólise e a espondilolistese são comuns. Nos atletas adolescentes a espondilólise é responsável por 47\% das dores lombares enquanto que nos adultos $5 \%{ }^{(11)}$. Por outro lado, a lombalgia é o principal sintoma da espondilólise embora em muitos casos ela seja diagnosticada como achado ocasional nos exames de imagens ${ }^{(1,4,9,12)}$.

Em atletas competitivos a incidência de lombalgia pode chegar a $100 \%{ }^{(9)}$. Entre atletas olímpicos, encontraram-se 63\% de ginastas com alterações constatáveis ao exame de ressonância magnética ${ }^{(4)}$, comprovando-se que, quanto mais longo e exaustivo o treinamento, maior será a sobrecarga na coluna vertebral que resultará em lesão nesse segmento ${ }^{(4,6-9,12,13)}$.

O objetivo deste trabalho é determinar a incidência de espondilólise e espondilolistese em atletas de ginástica artística feminina e sua correlação com dor lombar, história de trauma e carga de treinamento.

\section{MÉTODOS}

Avaliação voluntária de 18 atletas femininas de ginástica olímpica em nível competitivo de oito a 17 anos, provenientes da equipe do Centro Olímpico de Treinamento e Pesquisa (COTP), utilizando-se como critério de inclusão:

- Ser praticante de ginástica olímpica regular há pelo menos um ano.

- Apresentar boa saúde física e mental.

- Ter entre oito e 17 anos de idade no momento da pesquisa.

- Ser voluntária.

- Ter um responsável legal informado, lido, concordado e assinado o termo de consentimento livre e esclarecido.

Os critérios de exclusão foram:

- Apresentar no momento do exame processo infeccioso ou tumoral agudo.

- Ter história de cirurgia recente.

- Apresentar história de hipertensão arterial sistêmica não controlada.

- Ter histórico de distúrbios neurológicos sistêmicos e/ou convulsões.
- Apresentar no dia do exame frequência cardíaca acima de 100 batimentos por minuto ou pressão arterial acima de 160 x 100mmHg, em repouso.

Em todas as atletas foram realizados anamnese e exame físico dirigidos, investigando queixas lombares e alterações desse segmento ao exame clínico, seguidos do preenchimento de um questionário sobre suas atividades esportivas e eventuais lesões em decorrência do treinamento. Em seguida, as atletas foram submetidas à avaliação radiográfica nas incidências posteroanterior e perfil de coluna toracolombossacra em posição ortostática com aparelho de radiografia Siemens ${ }^{\circledR}$ ajustado para o potencial de 70KV. Os exames radiológicos foram avaliados num primeiro momento por ortopedistas especialistas em cirurgia de coluna vertebral, os quais não tiveram contato algum com as atletas. A seguir confrontaram-se os dados da história clínica de cada atleta com os achados radiográficos.

\section{RESULTADOS}

A idade media das atletas foi de 11,38 anos (oito a 17 anos) e o índice de massa corpórea (IMC) de 16,84 em média.

A investigação do padrão de dominância dos membros apresentado revelou 14 atletas destras $(77,77 \%)$, três ambidestras (16,6\%) e uma canhota (5,5\%) (Tabela 1).

Tabela 1 - Composição corporal das atletas da amostra

\begin{tabular}{c|c|c|c|c|c}
\hline Atleta & Idade & Peso & Altura & IMC & Dominância \\
\hline 1 & 14 & 35 & 1,5 & 15,55 & $\mathrm{D}$ \\
\hline 2 & 13 & 42 & 1,53 & 17,94 & $\mathrm{D}$ \\
\hline 3 & 8 & 35 & 1,45 & 16,64 & $\mathrm{D}$ \\
\hline 4 & 10 & 26 & 1,3 & 15,38 & $\mathrm{D}$ \\
\hline 5 & 13 & 35 & 1,42 & 17,35 & $\mathrm{~A}$ \\
\hline 6 & 10 & 27 & 1,32 & 15,49 & $\mathrm{D}$ \\
\hline 7 & 9 & 26 & 1,28 & 15,86 & $\mathrm{D}$ \\
\hline 8 & 13 & 36 & 1,6 & 14,06 & $\mathrm{D}$ \\
\hline 9 & 11 & 30 & 1,31 & 17,48 & $\mathrm{D}$ \\
\hline 10 & 17 & 32 & 1,39 & 16,56 & $\mathrm{D}$ \\
\hline 11 & 12 & 27 & 1,21 & 18,44 & $\mathrm{D}$ \\
\hline 12 & 8 & 34 & 1,34 & 18,93 & $\mathrm{~A}$ \\
\hline 13 & 10 & 26 & 1,33 & 14,69 & $\mathrm{D}$ \\
\hline 14 & 11 & 27 & 1,3 & 15,97 & $\mathrm{D}$ \\
\hline 15 & 12 & 45 & 1,54 & 18,97 & $\mathrm{~A}$ \\
\hline 16 & 11 & 47 & 1,54 & 19,81 & $\mathrm{E}$ \\
\hline 17 & 14 & 23 & 1,21 & 15,71 & $\mathrm{D}$ \\
\hline 18 & 9 & 42 & 1,51 & 18,42 & $\mathrm{D}$ \\
\hline
\end{tabular}

Idade em anos; peso em quilogramas; altura em metros; IMC- índice de massa corpórea; dominância: A- ambidestra, D- destra, E-canhota 
Quanto à intensidade do treinamento, as ginastas treinaram em média 27,77 horas por semana; 13 atletas (72,22\%) dedicam-se aos quatro aparelhos da modalidade, três (16,66\%) restringiam o treinamento aos exercícios de solo, barra e trave; uma atleta $(5,5 \%)$ dedica-se aos exercícios de barra assimétrica e trave de equilíbrio e uma (5,5\%) atleta atém-se só aos exercícios de solo. As atletas que treinam um número inferior de horas por semana o fazem devido a lesões e tratamentos fisioterápicos realizados no horário do treino dentro do mesmo complexo esportivo.

O tempo de dedicação ao esporte competitivo variou de um a oito anos, média de 4,27 anos (Tabela 2).

Tabela 2 - Estrutura de treinos realizados pelas ginastas

\begin{tabular}{c|c|c}
\hline Estrutura de treino & & Atletas \\
\hline \multirow{4}{*}{ h/sem } & $10 \mathrm{~h}$ & 1 \\
\cline { 2 - 3 } & $20-25 \mathrm{~h}$ & 3 \\
\cline { 2 - 3 } & $30 \mathrm{~h}$ & 14 \\
\hline \multirow{4}{*}{ Aparelhos } & Solo & 1 \\
\cline { 2 - 3 } & Barra e trave & 1 \\
\cline { 2 - 3 } & Solo, barra e trave & 3 \\
\cline { 2 - 3 } Compete & Todos & 13 \\
\cline { 2 - 3 } & Até 2 anos & 4 \\
\cline { 2 - 3 } & $2-5$ anos & 9 \\
\hline \multirow{4}{*}{} & Mais de 5 anos & 5 \\
\hline
\end{tabular}

$\mathrm{h} / \mathrm{sem}$ : horas dedicadas ao treinamento por semana. Compete: tempo (em anos) em que participa de competições oficiais.

A saúde geral de 14 (77,77\%) das atletas da amostra é excelente. Uma atleta (5,5\%) possui o quadro de sinusite alérgica controlado com medidas gerais. As três ginastas (16,6\%) restantes possuíam agravos à saúde que necessitam uso de medicação de uso contínuo. Porém, o uso da medicação não interferiu na qualidade de treinamento dessas atletas.

A pesquisa de antecedentes mórbidos pessoais indicou 15 atletas $(83,33 \%)$ isentas de procedimentos cirúrgicos prévios. Duas atletas $(11,1 \%)$ foram submetidas à herniorrafia umbilical na primeira infância e uma atleta (5,5\%) sofreu fratura fechada de cotovelo esquerdo em 2005 que necessitou de procedimento cirúrgico.

No questionário específico sobre condições físicas relacionadas com o treinamento, 15 atletas referiram dores limitantes em diferentes locais, frequências e intensidades.

Às questões com relação à presença de dores, independente da localização, nove atletas (50\%) referi- ram dores em todos os treinos; quatro (22,22\%), dores frequentes; duas $(11,11 \%)$, dores ocasionais; e três (16,66\%) negam prejuízos no treinamento devido à dor (Tabela 3). Entendemos por "sempre” uma periodicidade de quatro ou cinco episódios de dor a cada semana; "frequente" refere-se ao aparecimento de episódio doloroso entre duas e três vezes por semana; "ocasional” ou “às vezes”, quando a dor se dá cerca de uma vez por semana; quando a atleta relata dor com a frequência de uma vez ao mês foi denominado "raramente" e "desprezível” quando a aponta episódios pontuais de dor.

Quando especificada a região lombar como sede de dor limitante, três ginastas $(16,66 \%)$ referiram dor em todos os treinos, três atletas $(16,66 \%)$ com dores frequentes, cinco ginastas $(27,77 \%)$ relataram dor lombar ocasional, quatro $(22,22 \%)$ referiram dor lombar em raras ocasiões e três (16,66\%) não apontaram a coluna como sede importante de dor (Tabela 3).

Tabela 3 - Dor relacionada com o treinamento

\begin{tabular}{c|c|c}
\hline & $\begin{array}{c}\text { Atletas com dor nos } \\
\text { treinos }\end{array}$ & Atletas com dor lombar \\
\hline Sempre & 9 & 3 \\
\hline Frequente & 4 & 3 \\
\hline Às vezes & 2 & 5 \\
\hline Raramente & 0 & 4 \\
\hline Desprezível & 3 & 3 \\
\hline
\end{tabular}

Ao se relacionar o aparecimento da dor com o período de treinamento, em 13 (72,22\%) casos o pico da dor ocorreu durante o treinamento, duas atletas $(11,1 \%)$ disseram sentir dor logo após o treino e três atletas (16,66\%) não relacionaram o aparecimento da dor com o treinamento imediato (Tabela 4).

A intensidade da dor é um parâmetro muito subjetivo. Para fim de estudo comparativo usou-se o número de competições esportivas das quais as atletas deixaram de participar devido a dores. Os resultados revelaram que 12 ginastas $(66,66 \%)$ nunca deixaram de competir devido às dores e lesões decorrentes de treinamentos. Em contraposição, três atletas (16,66\%) deixaram de participar de um campeonato importante devido à dor. Uma atleta (5,5\%), pelo quadro álgico, deixou de comparecer à sua apresentação em duas ocasiões, outra (5,5\%) deixou de participar de cinco competições e, por fim, uma atleta (5,5\%) absteve-se inúmeras vezes de eventos esportivos devido à dor relacionada com treinamento e lesões esportivas de pequena complexidade (Tabela 4). 
Tabela 4 - Intensidade e aparecimento da dor

\begin{tabular}{c|c|c}
\hline \multirow{4}{*}{ Deixou de competir } & Não & Atletas \\
\hline & 1 vez & 12 \\
\cline { 2 - 3 } & 2 vezes & 3 \\
\cline { 2 - 3 } & 5 vezes & 1 \\
\cline { 2 - 3 } & Diversas vezes & 1 \\
\hline \multirow{3}{*}{ Treino X dor } & Durante o treino & 1 \\
\cline { 2 - 3 } & Após o treino & 14 \\
\cline { 2 - 3 } & Sem relação & 2 \\
\hline
\end{tabular}

Deixou de competir: por quantas vezes deixou de participar de eventos oficiais nos quais estava classificada, porém não competiu devido a restrições médicas. Treino $\mathrm{X}$ dor: comparativo de relação temporal do treino e do aparecimento da dor.

A seguir, foi realizada avaliação radiográfica das atletas com ênfase na determinação dos parâmetros anatômicos e patológicos, resumidos na Tabela 5. O ângulo de lordose no exame radiográfico variou de $10^{\circ}$ a $40^{\circ}$, média de $23^{\circ} 83^{\prime}$ pelo método de Cobb (linha tangente ao platô superior de L1 e linha inferior da superfície articular de L5; ângulo de lordose é igual ao ângulo formado pelo cruzamento das perpendiculares dessas linhas). O ângulo sacral situou-se entre $20^{\circ}$ e $55^{\circ}$, com média de $37^{\circ} 33^{\prime}$. A incidência sacral esteve dentro de $5^{\circ}$ a $32^{\circ}$ (média: $22^{\circ} 50$ '). A obtenção dessa medida foi feita a partir de uma linha tangente à borda posterior do sacro em cruzamento com uma linha perpendicular ao solo. Outro fato anatômico relevante identificado nas radiografias foi a incidência de espinha bífida. No total, oito $(44,45 \%)$ casos radiograficamente foram apontados, sendo sete $(38,88 \%)$ em S1 e um $(44,4 \%)$ em L5.

A pesquisa para espondilólise resultou no encontro de um caso (5,5\%) na $5^{a}$ vértebra lombar (Figura 1 ). Não foi identificado caso de espondilolistese na amostra

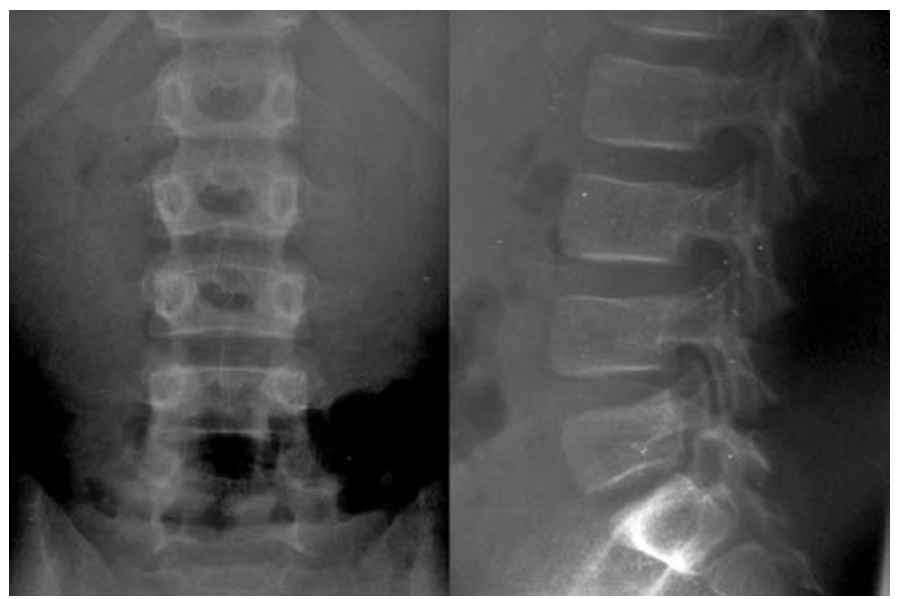

Figura 1 - Radiografia frente e perfil da coluna lombossacra evidenciando a espondilólise de L5 estudada. A atleta que apresentou sinais de espondilólise tem oito anos de idade, compete nos quatro aparelhos há um ano e não possui alterações clínicas. Treina cerca de 20 horas semanais sem restrições. Dentro do grupo ela é uma das atletas menos queixosas em relação às dores nos treinos, tanto lombares como em outras regiões do corpo (Tabela 5).

Tabela 5 - Dados radiográficos

\begin{tabular}{c|c|c}
\hline Achados radiográficos & & Atletas \\
\hline Espondilólise & L5 & 17 \\
\hline Espinha bífida & Ausente & 1 \\
\hline & L5 & 7 \\
\hline Lordose L1-L5 & S1 & 10 \\
\hline & Ausente & 8 \\
\hline Angulo sacral & $10-20^{\circ}$ & 4 \\
\hline & $21-30^{\circ}$ & 6 \\
\hline
\end{tabular}

\section{DISCUSSÃO}

Tendo em vista que a lombalgia é a principal queixa de consulta ao ortopedista geral, é importante conhecer a sua etiologia. Com importante prevalência dentre essas causas, estão a espondilólise e a espondilolistese $^{(4,9,10,11)}$.

O sintoma prevalente de ambas é a dor lombar, sendo pior com as atividades físicas, hiperextensão lombar e após tempo prolongado na mesma posição. Pode haver irradiação para nádegas e região posterolateral da coxa, parestesia, claudicação e sintomas compressivos radicular ou até mesmo medular ${ }^{(10)}$.

Estudos da literatura mostram prevalência de 4,4\% a 5\% para espondilólise em crianças de idade escolar, chegando a 6\% em torno dos 14 anos e 7,2\% em $\operatorname{adultos}^{(14,15)}$. Constatou-se, ainda, maior prevalência no sexo masculino bem como nos esquimós do Alasca, e menor em negros ${ }^{(16)}$. Entre os fatores etiológicos, sabese que a hereditariedade é determinante, assim como o crescimento $^{(2)}$. A presença de traumatismo é considerado fator causal, o mesmo ocorrendo com microlesões de repetição ${ }^{(12)}$. Conforme sugerido na literatura, uma possível causa de espondilólise é fratura por estresse 
da pars interarticularis devido a microtraumas, algo que faria pressupor o aumento na prevalência em certos esportes que geram hiperextensão e, por conseguinte, contato da borda caudal da faceta articular inferior com a $\operatorname{pars}^{(9,11)}$. Dentre os esportes em questão citam-se os praticantes de levantamento de peso, jogadores de futebol, mergulhadores, bailarinas e ginastas ${ }^{(6,10)}$.

Os exames radiográficos foram analisados por ortopedistas especialistas em cirurgias de coluna, obtendo prevalência de 5,56\% para espondilólise e não havendo casos de espondilolistese.

Não foi observada, portanto, diferença entre a prevalência em ginastas e na população geral. Apesar de ser uma amostra pequena, o resultado obtido sugere não haver relação direta entre ginástica olímpica e espondilólise ou espondilolistese. Uma das possíveis causas para a ausência de lise e listese na coluna vertebral das ginastas é que, apesar da sobrecarga de hiperextensão e carga, o condicionamento muscular acima dos padrões habituais em alongamento e flexibilidade compensaria o estresse sofrido. No caso específico da listese, o fortalecimento muscular oriundo do próprio esporte e as sessões de alongamento às quais as atletas são submetidas preveniram sua ocorrência. Já em se tratando de espondilólise, o balanço entre fator causal e prevenção traz a prevalência para próxima da população não desportista.

\section{REFERÊNCIAS}

1. Cohen M, Abdalla R J. Ginástica olímpica. In: Lesões nos esportes. Diagnóstico prevenção e tratamento. São Paulo: Revinter; 2002. p.776-84.

2. Garcés GL, González-Montoro I, Rasines J L, Santoja F. Early diagnosis of stress facture of the lumbar spine in athletes. Int Orthop. 1999;23(4):213-5.

3. Kolt GS, Kirkby RJ. Epidemiology of injury in elite and subelite female gymnasts: a comparison of retrospective and prospective findings. $\mathrm{Br} \mathrm{J}$ Sports Med. 1999;33(5):312-8.

4. Tertti M, Paajanen H, Kujala UM, Alanen A, Salmi TT, Kormano M. Disc degeneration in young gymnasts. A magnetic resonance imaging study. Am J Sports Med. 1990;18(2):206-8.

5. Dixon M, Fricker P. Injuries to elite gymnasts over 10 yr. Med Sci Sports Exerc. 1993;25(12):1322-9.

6. Garrick James G, Requa Ralph K. Epidemiology of women's gymnastics injuries. Am J Sports Med. 1980;8(4):261-4.

7. Pettrone FA, Ricciardelli E. Gymnastic injuries: the Virginia experience 19821983. Am J Sports Med. 1987;15(1):59-62.

8. Snook GA. Injuries in women's gimnastics. Am J Sports Med. 1979;7(4):242-4.
Em vista do analisado, pressupõe-se que a ginástica olímpica não predispõe a lise ou listese da coluna toracolombossacra. Isso despertaria dúvidas quanto à sua ocorrência em outros esportes que apresentam hiperextensão e motiva a realizar estudos semelhantes em atletas de outras especialidades, como mergulho, levantamento de peso, paraquedismo e futebol americano.

É notório que devemos procurar outras causas de lombalgia nas atletas, bem como jamais poderemos negligenciar a preparação física em relação à preparação técnica, já que ela parece ser um fator decisivo na prevenção de lesões anatômicas na coluna lombar.

\section{CONCLUSÕES}

Concluímos que a incidência de espondilólise e espondilolistese foi pequena, na amostra, quando comparada com a literatura, e que não apresentou relação com a presença de dor lombar nas atletas que praticam ginástica artística. Os ângulos sacrais, da incidência sacral e da lordose lombar não apresentaram correlação com a presença de sintomas de dor ou espondilolistese. Convém salientar que o estudo apresenta uma amostra pequena de atletas, porém, a incidência de lesão aproxima-se da ocorrência em pacientes não atletas relatada na literatura.
9. Bozdech Z, Dufek P. Spondylolisthesis in young gymnasts. Acta Univ Carol Med (Praha). 1986;32(5-6):405-9.

10. Ibarra AG, Miñarro PAL. Espondilolisis y espondilolistesis en la práctica físicodeportiva. Revista digital- Buenos Aires [periódico na internet] 2003 [citado Jan 2003; 8(56)]. Disponível em http://efdeortes.com/.

11. Cassidy RC, Shaffer WO, Johnson DL. Spondylolysis and spondylolisthesis in the athlete. Orthopedics. 2005;28(11):1331-3.

12. Standaert CJ. Spondylolysis in the adolescent athlete. Clin J Sports Med. 2002;12(2):119-22.

13. Lowry C Benton, Leveau Barney F. A retrospective study of gymnastics injuries to competitors and noncompetitors in private clubs. Am J Sports Med. 1982;10(4):237-9.

14. Baker DR, McHollick W. Spondylolysis and spondylolisthesis in children. J Bone Joint Surg Am. 1978;38:933-4.

15. Frederickson BE, Baker D, McHolick WJ, Hansen AY, Luubicky JP. The natural history of spondylolysis and spondylolistesis. J Bone Joint Surg 1984;66(5):699-707.

16. Stewart TD. The age incidence of neural arch defects in Alaska natives, considered from standpoint of etiology. J Bone Joint Surg Am. 1953; 35(4):935-50. 